\title{
Raman Scattering and X-ray Diffraction Study of Neutron Irradiated GaN Epilayers
}

\author{
R. X. Wang ${ }^{1 *}$, S. J. Xu ${ }^{1}$, S. Li ${ }^{1}$, S. Fung ${ }^{1 \dagger}$, C. D. Beling ${ }^{1}$, K. Wang ${ }^{1}$, Z.F. Wei ${ }^{1}$, T.J. Zhou ${ }^{1}$ and J. D. Zhang ${ }^{1}$, M. Gong ${ }^{2}$, \\ G. K. H. Pang ${ }^{3}$ \\ ${ }^{1 *}$ Department of Physics, The University of Hong Kong, Pokfulam Road, Hong Kong, P. R. China \\ *email: wangrx@hkusua.hku.hk \\ †email: sfung@hkucc.hku.hk \\ Tel: (852)-28592374, Fax: (852)-28557445 \\ ${ }^{2}$ Department of Physics, Sichuan University, Chengdu, P. R. China \\ ${ }^{3}$ Department of Physics, The Hong Kong Polytechnic University, Hung Hom, Kowloon, Hong Kong, P. R. China
}

\begin{abstract}
Neutron irradiation induced defects and their effects on the carrier concentration of GaN epilayers are investigated with Raman scattering and $\mathrm{X}$-ray diffraction techniques. Relative to the as-grown sample, the neutronirradiated samples exhibit a clear variation in the position and lineshape of the $A_{1}(\mathrm{LO})$-mode Raman peak as well as in the fullwidth at half-maximum height (FWHM) of the XRD rocking curves. Careful curve fitting and adequate calculations give the carrier concentrations of the irradiated GaN. It is found that the defects induced by neutron irradiation act as carrier trap centres which capture the electron carriers so that the carrier concentration of the irradiated $\mathrm{GaN}$ is reduced.
\end{abstract}

\section{Keywords: GaN, neutron irradiation, defects}

\section{INTRODUCTION}

Gallium nitride $(\mathrm{GaN})$ is currently a material attracting technological interest [1-3] due to its application in blue/ultraviolet light emitting devices[4,5], sun blind detectors [6] and high power/high temperature electronics. Although $\mathrm{GaN}$ based light emitting devices including laser diodes have been commercialized, the effects of various defects on device performance are still far from being fully understood. Moreover, the stability and reliability of devices which operate in radiation environments is also an important area of study. It is well known that irradiation by high-energy particles such as neutrons and electrons can induce structural defects in semiconductors. In the past few decades, there have been many reports discussing the irradiation-induced defects in silicon [7], GaAs [8-10], GaP [11] and InP [12]. However, only a few studies $[13,14]$ have reported on the effects of neutron irradiation on the microstructural and optical properties of $\mathrm{GaN}$ thin films. In this work neutron irradiation induced defects and their effects on the carrier concentration of $\mathrm{GaN}$ epilayers have been investigated using Raman scattering and X-ray diffraction $\omega$ rocking scan curve (XRD) techniques. It is found that the neutron-irradiated samples exhibit a clear variation in the position and lineshape of the $\mathrm{A}_{1}(\mathrm{LO})$-mode Raman peak as well as in the full-width at half-maximum height (FWHM) of the XRD spectra as the neutron irradiation dosage increases. Careful curve fitting to the $\mathrm{A}_{1}(\mathrm{LO})$-mode Raman peak using theoretical modeling based on the plasmon frequency is used to determine the carrier concentrations of the neutron-irradiated GaN. A reduced carrier concentration is observed in the irradiated material which is explained on the basis of structural damage/or defects induced by the neutron irradiation that act as carrier trapping centers. The trap centres act to compensate the carrier concentration in $\mathrm{GaN}$.

\section{EXPERIMENTS}

The GaN wafer used in the study was grown on a sapphire (0001) substrate using hydride vapor phase epitaxy (HVPE) method. The GaN had a room temperature carrier concentration and mobility of $3.3 \times 10^{17} \mathrm{~cm}^{-3}$ and $258 \mathrm{~cm}^{2} \mathrm{~V}^{-1} \mathrm{~s}^{-1}$ respectively as measured by Hall measurement. To study the effect of neutron radiation on $\mathrm{GaN}$, the wafer was cut into small pieces with area $4 \mathrm{~mm} \times 4 \mathrm{~mm}$. Some pieces of the sample were irradiated by neutrons $(30 \%$ ratio of fast neutrons to thermal neutrons) under different dosages, i.e, $1 \times 10^{15}$, $5 \times 10^{15}$, and $1 \times 10^{16} \mathrm{~cm}^{-2}$. In the following these three samples are referred to as "low", "middle" and "high" -dosage, respectively with the as-grown sample being used as a reference. The irradiated samples were not annealed.

The crystal structures of the samples were investigated using XRD $\omega$ rocking scans. The measurement system is a high intensity $\mathrm{Cu} \mathrm{K} \alpha$ Bruker D8 Discover X-ray diffractometer with a divergence of less than $0.05^{\circ}$ at $0.001 /$ step speed. The micro-Raman scattering experiments were carried out under a backscattering geometry with a combination of instruments, namely a monochromator equipped with a 1800 lines $/ \mathrm{mm}$ grating, a microscope, a charge coupled device detector cooled by liquid nitrogen and a notch filter. The $488 \mathrm{~nm}$ line of a Coherent $\mathrm{Ar}^{+}+\mathrm{Kr}^{+}$mixed gas laser was employed as the excitation light in the Raman measurements. The typical spatial resolution of the Raman instrument is about $1 \mu \mathrm{m}$. The detailed description of the confocal micro-Raman system can be found in a previous publication [15]. All Raman scattering and XRD experiments were performed at room temperature. 


\section{RESULTS AND DISCUSSION}

Figure 1 shows the measured micro-Raman spectra of the $\mathrm{GaN}$ irradiated by different dosages of neutrons. The intensity

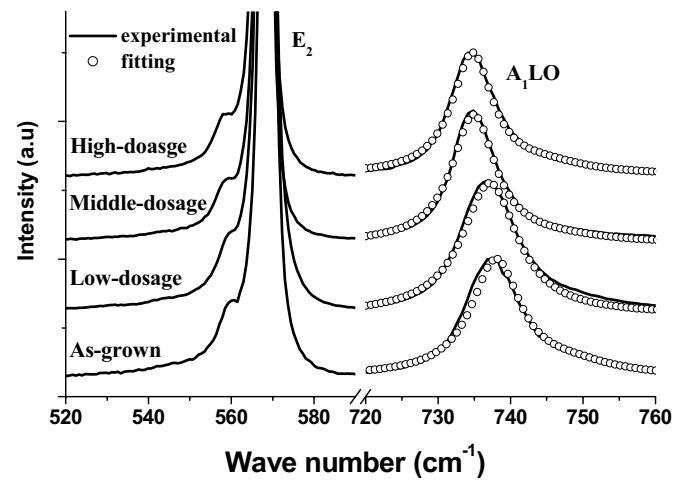

Fig.1 Micro-Raman spectra of the GaN as-grown and irradiated by neutron with different dosages

of the $A_{1}(L O)$ peak in each curve is normalized to unity. For clarity, the curves are vertically shifted. From the figure, it can be seen that the $A_{1}(\mathrm{LO})$ mode of the neutron-irradiated $\mathrm{GaN}$ samples shifts towards the low-frequency side. Moreover, the lineshape and FWHM of the $\mathrm{A}_{1}(\mathrm{LO})$ mode also change with neutron dosage relative to the as-grown sample. By comparison the $E_{2}$ (high) mode of all the samples including as-grown one does not change, confirming that the $\mathrm{A}_{1}(\mathrm{LO})$ mode's redshift seen in the irradiated samples is not due to any systematic experimental effect. It is known that the plasma mode of a free electron gas can couple with the LO phonon lattice vibration mode in semiconductors giving rise to the so-called coupled mode whose frequency is closely related to the electron density $[1,3,16,17]$. In order to estimate the free electron density in the neutron-irradiated GaN samples the $A_{1}(L O)$ modes peak shape was fitted to the functional form[17-20]:

$$
I(\omega)=K A(\omega) \operatorname{Im}\left(-\frac{1}{\varepsilon_{\infty}\left[1+\frac{\omega_{L}^{2}-\omega_{T}^{2}}{\omega_{T}^{2}-\omega^{2}-i \omega \Gamma}-\frac{\omega_{p}^{2}}{\omega(\omega+i \gamma)}\right]}\right)
$$

where

$$
\begin{aligned}
& A(\omega)=1+2 C \frac{\omega_{T}^{2}}{\Delta}\left[\omega_{p}^{2} \gamma\left(\omega_{T}^{2}-\omega^{2}\right)-\omega^{2} \Gamma\left(\omega^{2}+\gamma^{2}-\omega_{p}^{2}\right)\right]+ \\
& C^{2}\left(\frac{\omega_{T}^{4}}{\Delta\left(\omega_{L}^{2}-\omega_{T}^{2}\right)}\right)\left\{\omega_{p}^{2}\left[\gamma\left(\omega_{L}^{2}-\omega_{T}^{2}\right)+\Gamma\left(\omega_{p}^{2}-2 \omega^{2}\right)\right]+\omega^{2} \Gamma\left(\omega_{p}^{2}+\gamma^{2}\right)\right\}
\end{aligned}
$$

and where $\Delta$

$$
\Delta=\omega_{p}^{2} \gamma\left[\left(\omega_{T}^{2}-\omega^{2}\right)^{2}+(\omega \Gamma)^{2}\right]+\omega^{2} \Gamma\left(\omega_{L}^{2}-\omega_{T}^{2}\right)\left(\omega^{2}+\gamma^{2}\right)
$$

and the plasma frequency $\omega_{P}$ are given by

$$
\omega_{P}=\sqrt{\frac{4 \pi n e^{2}}{m^{*} \varepsilon \varepsilon_{0}}}
$$

where $\omega_{L}$ and $\omega_{T}$ are respectively the frequencies of the longitudinal and transverse optical phonons. $K, n, \gamma, \Gamma$ are respectively a constant coefficient, the carrier density, the plasmon damping constant and the phonon damping constant. Here we use $\omega_{T}=533 \mathrm{~cm}^{-1}, \omega_{L}=735 \mathrm{~cm}^{-1}, \varepsilon=5.35$ [1], $m^{*}=0.2 m_{0}$ [2] and $\mathrm{C}=0.4$ [18] for GaN. The fitting parameters are listed in Table I. The carrier concentrations of the samples derived are thus $2.5 \times 10^{17} \mathrm{~cm}^{-3}, 1.3 \times 10^{17} \mathrm{~cm}^{-3}$, $1.2 \times 10^{17} \mathrm{~cm}^{-3}$, and $2.9 \times 10^{17} \mathrm{~cm}^{-3}$ for the low-dosage, middledosage, high-dosage, and as-grown samples, respectively. Since GaN is transparent to the $488 \mathrm{~nm}$ excitation light used in the Raman measurements, the Raman signal from the sapphire substrate $\left(\sim 749 \mathrm{~cm}^{-1}\right)$ is observed and is taken into account in the fitting calculations [18].

Usually, carrier removal is mainly due to the trapping of majority carriers by structural defects $[8,9,20,21]$. To test this idea and to investigate the crystal structure of the irradiated GaN, XRD $\omega$ scans of the samples were made. Figure 2 shows the XRD $\omega$ scan spectra of the samples. From Fig. 2 and Table I, it can clearly be seen that the FWHM of the irradiated samples varies with irradiation dosage. It is noted that the FWHM of the as-grown sample's spectrum is a little larger compared to that of the low-dosage sample. This effect may, however, be due to the as-grown sample being taken from $\mathrm{GaN}$ closer the wafer edge. It is known that the quality of samples close to the edge is worse than for samples close to the center. Moreover, it is known that the FWHM of an XRD $\omega$ scan spectrum reflects the quality of a sample [22]. For a group of samples with the same crystal structure and chemical composition, the wider FWHM indicates a worsening of crystalline quality. Thus, from the XRD data, it can be concluded that the structural quality of the samples becomes worse after the GaN samples are irradiated by neutrons. During the irradiation process, the GaN structure is not only bombarded by thermal neutrons that transmute ${ }^{69} \mathrm{Ga},{ }^{71} \mathrm{Ga}$, ${ }^{72} \mathrm{Ga}$ to ${ }^{70} \mathrm{Ge},{ }^{72} \mathrm{Ge}$ and $/$ or ${ }^{14} \mathrm{~N},{ }^{15} \mathrm{~N}$ to ${ }^{16} \mathrm{O}[13]$ but also with fast neutrons that elastically collide with atomic nuclei to produce point defects. Both of these processes cause the crystal structure to slightly distort with a subsequent production of strain $[8,10,23]$. These defects can be shallow traps and/or deep level defects and even complex defect centers $[13,14,23]$. They generally act as carrier trapping centers and thus influence the carrier concentration of the neutron irradiated GaN.

\section{CONCLUSIONS}

GaN samples irradiated by different neutron dosages have been investigated using Raman scattering and XRD spectra techniques. It has been observed that the $\mathrm{A}_{1}(\mathrm{LO})$ peak position in the Raman scattering spectra clearly shifts towards the lower frequency side and the FWHM of XRD spectra 
TABLE I. Values of the fitting parameter for Raman $A_{1}(L O)$ mode and XRD FWHM

\begin{tabular}{|c|c|c|c|c|c|}
\hline \multicolumn{2}{|c|}{ Irradiation dosage } & As-grown & $1 \times 10^{15}$ & $5 \times 10^{15}$ & $1 \times 10^{16}$ \\
\hline \multirow{2}{*}{$\begin{array}{c}\text { Fitting } \\
\text { parameters }\end{array}$} & $\omega_{\mathrm{P}}$ & 120 & 105 & 55 & 50 \\
\cline { 2 - 6 } & $\gamma$ & 300 & 265 & 259 & 218 \\
\cline { 2 - 6 } Derived & $\Gamma$ & 5.4 & 7.1 & 7.0 & 6.8 \\
\hline \multicolumn{2}{|c|}{$\begin{array}{c}\text { Carrier } \\
\text { concentration } \\
\left(\times 10^{17} \mathrm{~cm}^{-3}\right)\end{array}$} & 2.9 & 2.5 & 1.3 & 1.2 \\
\hline \multicolumn{2}{|r|}{ XRD FWHM $\left(^{\circ}\right)$} & 0.106 & 0.103 & 0.112 & 0.109 \\
\hline
\end{tabular}

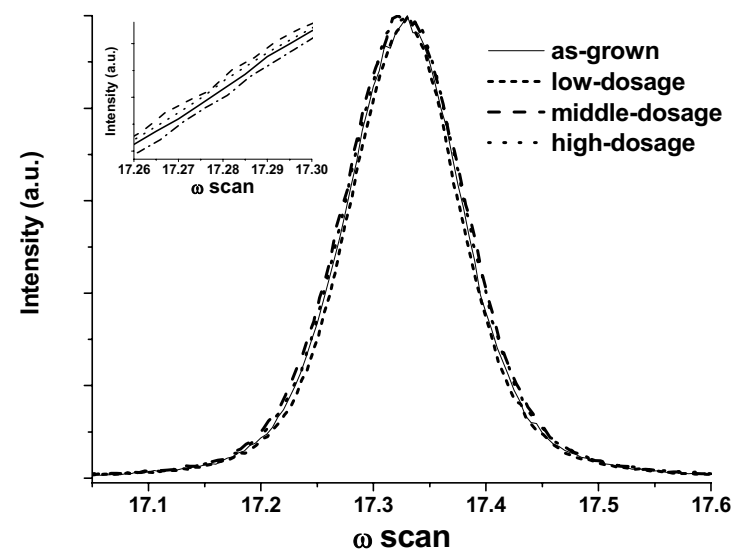

Fig.2 XRD spectra of GaN samples as-grown and irradiated by neutron with various dosages

generally increases with neutron irradiation dosage. From careful analysis of the experimental Raman spectra, it is found that the carrier concentration of $\mathrm{GaN}$ samples varies as the various neutron irradiation dosages. This has been explained on the bases of the structural damage and/or defects induced by neutron irradiation acting as carrier trapping centers which compensate the carrier concentration of the GaN samples.

\section{ACKNOWLEDGEMENTS}

We acknowledge partial support of the grant (HKU 7021/04P) from the Research Grant Council of Hong Kong. M. Gong also acknowledges support from the grant of national nature science of China (No. 60076010) and the grant of the National Key Lab of Silicon Materials of China.

\section{REFERENCES}

[1] H. Harima, H. Sakashita, T. Inoue, and S. Nakashima, "Electronic properties in doped GaN studied by Raman scattering", Journal of Crystal Growth, vol 189/190, pp.672-676, June 1998

[2] N.Wieser, M.Klose, R.Dassow, F.Scholz and J.Off, "Raman studies of longitudinal optical phonon-plasmon coupling in GaN layers", Journal of Crystal Growth, vol 189/190, pp.661-665, June 1998

[3] D.Meister, M.Böhm, M.Topf, W.Kriegseis, W.Burkhardt, I.Dirntorfer, S.Rösel, B.Frangis, B.K.Myer, A.Hoffmann, H.Siegle, C.Thomsen, J.Christen and F.Bertram, "A comparison of the Hall-effect and secondary ion mass spectroscopy on the shallow oxygen donor in unintentionally doped GaN films", J. Appl. Phys., vol 88, pp.18111817, August 2000

[4] H.X. Wang, H.D. Li, Y.B. Lee, H. Sato, K. Yamashita, T. Sugahara, S. Sakai, "Fabrication of high-performance $370 \mathrm{~nm}$ ultraviolet lightemitting diodes", Journal of Crystal Growth, vol 264, pp.48-52, March 2004

[5] C.F. Chu, F.I. Lai, J.T. Chu, C.C. Yu, C.F. Lin, H.C. Kuo, S.C. Wang, "Study of GaN light-emitting diodes fabricated by laser lift-off technique", J. Appl. Phys., vol 95, pp.3916-3922, April 2004

[6] J.A. Chediak, Z.S. Luo, J.G. Seo, N. Cheung, L.P. Lee, T.D. Sands, "Heterogeneous integration of CdS filters with GaN LEDs for fluorescence detection Microsystems", Sensors and Actuators APhysical, vol 111, pp.1-7, March 2004

[7] M.Coeck, N.Balcaen, T.Van Hoecke, B.Van Waeyenberge, D.Segers, C.Dauwe and C.Laermana, "Defects in neutron transmutation doped silicon studied by positron annihilation lifetime measurements", J. Appl. Phys., vol 87, pp.3674-3677, April 2000

[8] J. G. Williams, J. U. Patel, A. M. Ougouag and S. Y. Yang, "Carrier removal and change in electrical properties of neutron irradiated GaAs", J. Appl. Phys., vol 70, pp.4931-4937, November 1991

[9] G.E.Brehm and G.L.Pearson, "Gamma-radiation damage in epitaxial gallium arsenide”, J. Appl. Phys., vol 43, pp.568-573, February 1972

[10] M.Satoh, K.Kuriyama and T.Kawakubo, "Depth uniformity of electrical properties and doping limitation in neutron-transmutation-doped semiinsulating GaAs", J. Appl. Phys., vol 67, pp.3542-3544, April 1990

[11] M.Palczewska, J.Jasinski,K.Korona,M.Kaminska, E.D.Bourrent and A.G.Elliot, "Antisite defects created in neutron irradiated GaP crystals", J. Appl. Phys., vol 78, pp.3680-3685, September 1995

[12] B.Mari, M.A.Hernandez-Fenollosa, F.J.Navarro, "Observation of Ferelated defects in neutron irradiated semi-insulating InP", J. Appl. Phys., vol 89, pp.7772-7776, June 2001

[13] K. Kuriyama, T. Tokumasu, Jun Takahashi, H. Kondo, and M. Okada, "Lattice distortions and the transmuted-Ge related luminescence in neutron-transmutation-doped GaN", Appl. Phys. Lett., vol 80, pp.33283330, May 2002

[14] C. W. Wang, "Neutron irradiation effect on radio-frequency magnetronsputtered $\mathrm{GaN}$ thin films and $\mathrm{Au} / \mathrm{GaN}$ schottky diodes", J. Vac.Sci. Technol. B, vol 20, pp.1821-1826, September 2002

[15] D. G. Zhao, S. J. Xu, M. H. Xie, S. Y. Tong, and H. Yang, "Stress and its effect on optical properties of GaN epilayers grown on $\mathrm{Si}(111), 6 \mathrm{H}-$ SiC(0001), and c-plane sapphire", Appl. Phys. Lett., vol 83, pp.677679, July 2003

[16] W. Gotz, N. M. Johnson, H. Amano, and I. Akasaki, "Deep level defects in n-type GaN", Appl. Phys. Lett., vol 65, pp.463-465, July 1994

[17] H. Harima, S. Nakashima, and T. Uemura, "Raman scattering from anisotropic LO-phonon-plasmon-coupled mode in n-type $4 \mathrm{H}$ - and $6 \mathrm{H}-$ SiC”, J. Appl. Phy., vol 78, pp.1996-2005, August 1995

[18] T.Kozawa, T.Kachi, H.Kno, Y.Taga and M.Hashimoto, "Raman scattering from LO phonon-plasmon coupled modes in gallium nitride", J.Appl.Phys., vol 75, pp.1098-1101, January 1994

[19] H.Harima, "Properties of GaN and related compounds studied by means of Raman scattering”, J. Phys. Condens. Matter, vol 14, pp.R967-R993, September 2002

[20] M.Senthil, R.Kesavamoorthy, P.Magudapathy, "Raman scattering studies on hydrogen ion-implanted GaN", Nucl. Instr. and Meth. in Phys. Res. B, vol 179, pp.193-199, 2001

[21] A. Castaldini, A.Cavallini and L.Polenta, "Deep levels and irradiation effects in n-GaN", J.Phys.Condens.Matter., vol 12, pp.10161-10167, December 2000

[22] J. Y. Shi, L. P. Yu, Y. Z. Wang, G. Y. Zhang, and H. Zhang, "Influence of different types of threading dislocations on the carrier mobility and photoluminescence in epitaxial GaN", Appl. Phys. Lett., vol 80, pp.2293-2295, April 2002

[23] P. Boguslawski and J. Bernholc, "Doping properties of C, Si, and $\mathrm{Ge}$ impurities in GaN and AIN", Physical Review B, vol 56, pp.9496-9505, October 1997 